Livraisons

d'Histoire

de l'Architecture

\section{Livraisons de l'histoire de l'architecture}

$37 \mid 2019$

L'architecture mise à l'examen

\title{
Les logiques d'examen au Conseil des bâtiments civils
}

The logics of the deliberations of Conseil des bâtiments civils (1795-1848)

Die Examenslogik beim Conseil des bâtiments civils (1795-1848)

\section{Emmanuel Château-Dutier}

\section{(2) OpenEdition}

Journals

\section{Édition électronique}

URL : http://journals.openedition.org/lha/1382

DOI : $10.4000 /$ /ha. 1382

ISSN : 1960-5994

Éditeur

Association Livraisons d'histoire de l'architecture - LHA

Édition imprimée

Date de publication : 15 juin 2019

Pagination : 49-58

ISSN : $1627-4970$

Référence électronique

Emmanuel Château-Dutier, "Les logiques d'examen au Conseil des bâtiments civils », Livraisons de I'histoire de l'architecture [En ligne], 37 | 2019, mis en ligne le 02 janvier 2021, consulté le 30 janvier 2021. URL : http://journals.openedition.org//ha/1382 ; DOI : https://doi.org/10.4000//ha.1382 


\section{LES LOGIQUES D'EXAMEN AU CONSEIL DES BÂTIMENTS CIVILS (1795-1848)}

Le conseil des bâtiments civils était une institution entièrement dédiée à l'examen de l'architecture. Ses membres formulaient des avis, à l'intention du ministre de l'Intérieur, sur tous les projets d'architecture publique projetés en France. À travers l'examen des projets architecturaux qui lui étaient soumis, le conseil des bâtiments civils exerçait une influence notable sur la nature architecturale des projets d'édifices qu'il révisait. La grande série continue des procès-verbaux des séances du conseil des bâtiments civils de 1795 à 1848, conservée dans la sous-série F21 des Archives nationales de France, offre au chercheur une source sans équivalent non seulement pour l'étude de l'architecture publique mais aussi pour l'étude du jugement architectural ${ }^{1}$.

Nous nous proposons avec cet article de nous intéresser au cœur même de la délibération du conseil des bâtiments civils. Après avoir examiné la question des prérogatives du Conseil, nous présenterons la procédure mise en œuvre par le Conseil pour l'examen des affaires afin de présenter quelques pistes méthodologiques pour aborder ce gigantesque matériau textuel dont l'étude nous semble permettre d'envisager, à nouveaux frais, la question de l'éclectisme en architecture.

\section{Les prérogatives du conseil des bâtiments civils}

Tout au long de la première moitié du dix-neuvième siècle, l'administration des bâtiments civils avait été chargée de la haute surveillance de la plupart des édifices construits sur les fonds publics à destination du gouvernement ou des autorités locales ${ }^{2}$. En 1795, le ministre de l'Intérieur Pierre Bénézech (1749-1802) avait voulu s'assurer qu'aucun ouvrage ne se fît sans que le gouvernement n'eût pu en faire examiner les plans et devis et en avoir autorisé l'exécution. Le principe de cet examen préalable se fondait principalement sur des règles de bonne gestion budgétaire. Celles-ci s'accompagnaient de mesures d'ensemble concernant l'organisation des agences d'architecture et le contrôle de la comptabilité des travaux afin de faciliter la vérification des mémoires de dépense concernant les bâtiments civils.

1. Les délibérations du conseil des bâtiments civils sont conservées aux Archives nationales de France au sein des registres cotés $\mathrm{F}^{21 *} 2470$ a 2542.

2. Emmanuel Château-Dutier, Le conseil des bâtiments civils et l'administration de l'architecture publique en France au XIX ${ }^{e}$ siècle, 1795-1848, Paris, thèse de doctorat de l'École pratique des hautes études, sous la direction de Jean-Michel Leniaud, 2016. 
Ce contrôle architectural portait sur trois objets. D'une part la nécessité des ouvrages dont l'exécution était proposée, c'est-à-dire la question de l'opportunité et de l'utilité des travaux. Le contrôle de leur bonne exécution et la conformité de la réalisation aux projets adoptés. Enfin, de la légitimité des paiements en acomptes ou des paiements définitifs. C'était pour remplir ces vues que le ministre de l'Intérieur avait pensé qu'il était convenable de s'entourer des lumières d'artistes, connus et expérimentés, en formant un conseil d'examen.

Telles que ses missions étaient présentées dans sa première organisation, le rôle du conseil des bâtiments civils relevait plus de la police architecturale que d'un véritable contrôle esthétique appliqué aux projets d'architecture ${ }^{3}$. Cependant, il ne faisait aucun doute que le Conseil aurait à s'occuper de toutes les questions d'art et de construction qui étaient soumises au ministre dans les attributions duquel il était placé.

L'organisation d'une commission consultative permettait aussi d'écarter en partie les accusations d'arbitraire. Créé au sortir de la Révolution, le conseil des bâtiments civils était, de ce point de vue, également en partie l'héritier des débats révolutionnaires sur le concours et l'accès démocratique à la commande publique ${ }^{4}$. Lucien Bonaparte (1775-1840) lorsqu'il fixa l'organisation du conseil des bâtiments civils, le $1^{\text {er }}$ vendémiaire an IX (23 septembre 1800) arrêta explicitement, que le conseil des bâtiments civils était chargé de la partie d'art des bâtiments ${ }^{5}$.

Les attributions du service des bâtiments civils et de son Conseil étaient particulièrement étendues, elles embrassaient l'ensemble des édifices projetés ou bâtis, soit pour le compte du Gouvernement, soit pour le compte de l'administration locale, y compris jusqu'au début de l'Empire les bâtiments qui étaient issus de l'ancienne Liste civile. Ce ne fut que par l'organisation de 1812 que ces attributions furent restreintes au seul ministère de l'Intérieur. Mais l'organisation de 1838, et celles qui suivirent mirent de nouveau les autres ministères à même de consulter le Conseil pour les différents travaux de leurs attributions. L'administration des bâtiments civils pouvait s'envisager, d'après l'expression de Jean Rondelet (1743-1829), comme une agence intermédiaire entre les architectes qui exécutaient les travaux et le gouvernement. Pour les membres du conseil des bâtiments civils, ce degré supplémentaire était destiné à y apporter de l'unité et une direction d'ensemble. Autrement dit, c'était en quelque sorte en architecte que le conseil des bâtiments civils pouvait envisager l'ensemble de l'équipement de la France et présider aux travaux.

3. Au sens de cette notion sous l'Ancien régime, il s'agissait de veiller au maintien de l'ordre social et de la bonne harmonie dans la polis, cf. Paolo Napoli, Naissance de la police moderne, Paris, La Découverte, 2003.

4. Werner Szambien, Les projets de l'an II. Concours d'architecture de la période révolutionnaire, Paris, École nationale supérieure de Paris, 1986.

5. Archives nationales de France (ci-après, Arch. nat.)., F ${ }^{21} 2474$, Procès-verbaux du conseil des bâtiments civils, "Nouvelle organisation du service des bâtiments civils [5 $5^{\mathrm{e}}$ organisation] " séance du 3 brumaire an IX (25 octobre 1800), p. 307-311. 
L'organisation mise en place en 1812 allait régir son fonctionnement pendant plus d'un quart de siècle, jusqu'en 1838. Son titre 2 détaillait les attributions qui lui étaient désormais dévolues, et son article 8 étendait ses attributions sous le rapport de l'art en précisant :

Outre l'examen des constructions sous le rapport des Beaux-Arts et des règles du goût, il examinera tout ce qui est relatif à la solidité des constructions, à la qualité des matériaux, aux convenances des édifices, tant sous le rapport de leur destination que des lieux où il s'agit de les construire. Il vérifiera les devis, s'assurera que rien n'y est omis, que tout y est justement calculé ; il se rendra compte des détails estimatifs, de leurs divers éléments, de la qualité des matériaux, de leurs prix aux lieux d'origine, des distances jusqu'aux lieux de construction; de l'espèce et du prix des transports, des prix des façons et mains-d'œuvre, etc. ${ }^{6}$

Les articles 9, 10, 11 et 12 ajoutaient encore que le Conseil émettrait toujours un avis sur les dépenses qu'il prévoyait et le soin avec lequel les projets étaient rédigés. Il était également chargé de donner son avis sur les honoraires qu’il était convenable d'allouer aux architectes d'après la qualité de leur travail ou leur participation aux travaux et les soins qu'ils avaient apportés à la solidité et l'économie des constructions. Continuant de s'occuper la rédaction du plan de Paris, il recevait dorénavant la possibilité de faire connaître son opinion sur les améliorations qui pourraient être apportées dans l'organisation des travaux publics pour obtenir de bons projets ou plus de garanties dans l'exécution. Enfin, le Conseil délibérait sur le contentieux des travaux, sur les demandes, prétentions et réclamations des entrepreneurs, des architectes, les questions de comptabilité et tous les objets que lui soumettrait le ministre. Le règlement sur les travaux du 22 juillet 1832, en précisant le rôle des inspecteurs généraux ${ }^{7}$ ajoutait explicitement à ces attributions l'examen des propositions des auteurs des découvertes nouvelles qui intéressaient le progrès de l'art en indiquant qu'ils feraient au besoin des expériences pour constater l'utilité des découvertes ${ }^{8}$.

Les attributions confiées au conseil des bâtiments civils par l'organisation de 1838 allaient considérablement étendre sa fonction puisqu'elle lui attribuait, rien de moins que le soin de «maintenir et de propager la bonne pratique de l'architec-

6. Arch. nat., $\mathrm{F}^{21}$ 2484, Procès-verbaux du conseil des bâtiments civils, "Arrêté du ministre concernant la nouvelle organisation du conseil des bâtiments civils ", séance du $1^{\text {er }}$ octobre 1812 , p. 1-7.

7. Arch. nat., $\mathrm{F}^{21}$ 6697, Arrêté du ministère du Commerce et des Travaux publics du 22 juillet 1832.

8. Emmanuel Château, "L'administration des bâtiments civils face aux questions constructives dans la première moitié du XIX ${ }^{\mathrm{e}}$ siècle en France ", Édifices et artifices, Histoires constructives, Robert Carvais, André Guillerme, Joël Sakarovitch et Valérie Nègre, éds., Paris, Picard, 2010, p. 365375. 
ture et de la construction ${ }^{9}$ ". Les arrêtés du 9 janvier $1840^{10}$ et du 20 décembre $1841^{11}$ laissaient inchangées les attributions du conseil des bâtiments civils qui continuait d'être régi par les titres II, III et IV de l'arrêté du 15 avril 1838 sauf une modification qui lui donnait la possibilité d'examiner des affaires qui lui étaient soumises par d'autres ministères.

Dans l'examen des projets d'architecture qui lui étaient présentés, le Conseil devait aussi s'assurer du respect d'un corpus de règles et de prescriptions concernant le domaine de la construction publique défini par un ensemble de lois, arrêtés et circulaires. De ce point de vue, on peut donc dire que, dans la première moitié du XIX ${ }^{\mathrm{e}}$ siècle, l'architecture publique constituait une pratique encadrée.

\section{La procédure d'examen}

La présentation du projet architectural au conseil des bâtiments civils exigeait la réunion d'un certain nombre de pièces dont le détail fut plus ou moins précisément prescrit selon les époques. Ce dossier était habituellement composé d'un devis détaillé et de pièces figurées établies d'après un code graphique fixé par voie réglementaire. On y adjoignit des plans de localisation, la fourniture du programme, et des documents concernant le mode d'exécution. Sous l'Empire, les circulaires du 22 octobre 1812 et l'Instruction du 28 juin 1813 allaient régler la composition des dossiers que devaient transmettre les préfets au ministère de l'Intérieur. Dans le service des travaux parisiens, le règlement sur les travaux de Paris du 13 mai 1824 prescrivait l'échelle des plans généraux et celle des profils et élévations de détails ${ }^{12}$ qui furent ensuite reconduites dans le règlement du 22 juillet $1832^{13}$.

L'organisation du 15 avril 1838 renouvelait la plupart de ces prescriptions sur la constitution des dossiers qui devaient être soumis au conseil des bâtiments civils ${ }^{14}$. Enfin, ces prescriptions furent développées dans l'arrêté du 20 décembre 1841 et surtout dans l'Instruction relative à la rédaction de tous les programmes, pro-

9. Arch. nat., $\mathrm{F}^{21}$ 2533, procès-verbaux du conseil des bâtiments civils, "Réorganisation du conseil des bâtiments civils [15 avril 1838] ", séance du 8 mai 1838, no 212 , p. 400-406. [Le texte du premier arrêté est transcrit dans Charles Pierre Gourlier, Notice historique sur le service des travaux des bâtiments civils à Paris et dans les départements, depuis la création de ce service en l'an IV (1795), Paris, L. Colas, 1848, Documents 1, p. 77-86.]

10. Arch. nat., $\mathrm{F}^{21}$ 924, Arrêté du ministre secrétaire d'État au département des Travaux publics du 9 juin 1840.

11. "Nouvelle organisation du conseil général des bâtiments civils ", Charles Pierre Gourlier, op. cit., Documents 3, p. 90 et suiv.

12. Arch. nat., $\mathrm{F}^{21} 924$, Instruction sur les fonctions des divers agents attachés au service des travaux du ministère de l'Intérieur et du département de la Seine, sous les ordres du directeur des Travaux publics de Paris, 13 mai 1824.

13. Arch. nat., $\mathrm{F}^{21}$ 6697, ministère du Commerce et des Travaux publics, 22 juillet 1832.

14. Arch. nat., $\mathrm{F}^{21}$ 2533, Procès-verbaux du conseil des bâtiments civils, "Réorganisation du conseil des bâtiments civils ", séance du 8 mai 1838, n 212, p. 400-406. [Le texte du premier arrêté est transcrit dans Charles Pierre Gourlier, op. cit., Documents 1, p. 77-86.] 
jets, devis, cahiers des charges, procès-verbaux, attachements, décomptes et autres pièces qui doivent être soumises à l'examen du conseil des bâtiments civils, ou qui en général ont pour objet le service des travaux des bâtiments civils à Paris et dans les départements du 15 avril 1842, renouvelée le $1^{\text {er }}$ février $1848^{15}$. Cette circulaire n'introduisait pas vraiment de dispositions nouvelles, mais elle avait le mérite de résumer les diverses dispositions en vigueur.

L'étude de la procédure administrative à partir des dossiers d'affaires permet d'envisager précisément les différents niveaux auxquels s'exerce le conseil dans l'élaboration du projet architectural. En fonction de la nature des édifices et selon les époques, le contrôle peut également concerner la réception des travaux. C'est ici qu'apparaît l'arme sans doute la plus efficace du service des bâtiments civils dans sa surveillance de l'architecture publique : la question du financement. Dans l'examen qu'il apportait aux dossiers qui lui étaient soumis, une part importante du travail du conseil des bâtiments civils consistait à juger de l'exactitude des devis. Les différentes règles apportées pour sa présentation étaient destinées à prévenir les dépassements dans la réalisation des ouvrages avec des pièces qui seraient par la suite utiles pour pouvoir procéder aux différents modes d'adjudication. Le contrôle économique des réalisations est constant, il répond aux règles de bonne gestion publique introduite par la Révolution, mais permet aussi de s'assurer de la conformité des réalisations avec les projets autorisés. Par ailleurs, l'obligation de se conformer à des procédures administratives strictes imposait un séquençage clair dans le processus de production des édifices entrepris pour le compte de l'État. Cette séquence implique une distinction nette entre les différentes étapes et le rôle de chacun des intervenants au cours de l'élaboration du projet, de la programmation, au déroulement du chantier et jusqu'à la liquidation des mémoires ou la réception de l'édifice qui ne fut pas sans conséquence sur l'organisation de la pratique.

\section{La délibération}

Les différents textes réglementaires concernant le fonctionnement du conseil des bâtiments civils fournissent assez peu d'informations sur son fonctionnement et notamment les modalités de la délibération collégiale. Le président était maître de l'ordre du jour et procédait à la répartition des affaires entre les membres pour la rédaction des rapports après un examen préalable en Conseil. Le projet de règlement pour la tenue des séances du conseil des bâtiments civils rédigé par Jean Rondelet (1743-1829) et Antoine-François Peyre (1739-1823) rend probablement

15. "Instruction relative à la rédaction de tous programmes, projets, devis, cahiers des charges, procèsverbaux, attachements, décomptes et autres pièces qui doivent être soumises à l'examen du conseil général des bâtiments civils, ou qui en général ont pour objet le service des travaux des bâtiments civils, à Paris et dans les départements, 15 avril 1842, et $1^{\text {er }}$ février 1848 ", dans Charles Pierre Gourlier, op. cit., p. 98-107. 
compte du fonctionnement du Conseil dans sa configuration étendue ${ }^{16}$. Il établissait un droit de présence qui était partagé entre les différents membres. L'assemblée avait alors lieu deux fois par décade, les duodi et tridi. En cas de besoin, le président pouvait proposer des assemblées particulières. Les séances s'ouvraient à midi et demi, le secrétaire donnait lecture de l'ordre du jour et faisait l'appel. On donnait ensuite lecture du procès-verbal de la séance précédente transcris sur le registre et celui-ci était signé par tous les membres après avoir été approuvé. Cette formalité était exigée avant qu'on puisse expédier n'importe quel extrait des avis ou les rapports. D'après l'organisation de 1800, les inspecteurs assistaient aux séances, mais ils n'avaient que voix consultative. Ils y rendaient compte de leur surveillance et des rapports qu'on leur avait confiés.

Le président procédait ensuite à la distribution des objets sur lesquels l'assemblée jugeait à propos qu'il lui soit fait un rapport. Le reste de la séance était employé à entendre la lecture des rapports sur les projets, à les discuter et prendre une décision sur chacun d'eux. Dans certains cas des personnes extérieures pouvaient être invitées à participer aux séances, après que le président ait exposé les motifs de cette invitation. Il n'était pas rare que le ministre vienne présider la séance lors des affaires importantes, et le responsable du service y assistait également fréquemment avec voix consultative pour y communiquer des informations.

Avec ses comptes rendus hebdomadaires sur ses opérations, la présidence de Jean-François Heurtier (1739-1822) donne l'occasion de comprendre un peu mieux les méthodes de travail des membres du conseil des bâtiments civils ${ }^{17}$. Il expliquait comment certaines affaires pouvaient bénéficier d'un examen sans rapport préalable pour accélérer la marche des affaires. Inversement, certaines affaires pouvaient occuper les discussions du Conseil pendant plusieurs séances, à raison de leurs difficultés et de leur complication. Ce fut notamment le cas de la voirie de Montfaucon, et des projets pour la couverture de la Halle aux blés, souvent aussi pour des projets de plans d'alignement importants. Dans certains cas, le renvoi à une commission, comme pour la Halle au Blé ou la Madeleine, permit de s'adjoindre des compétences extérieures. Dans les procès-verbaux, on trouve donc parfois la mention d'un examen préalable qui n'est pas transcrit au registre. Heurtier qui correspondait avec le ministre, accélérait les affaires, et exerçait, de fait, un réel ascendant sur ses collègues en tant que président.

À partir de 1812, ce fut le règlement du $1^{\text {er }}$ octobre qui régla les opérations. Celui-ci resta en vigueur jusqu'en 1838 . Il prescrivait un certain nombre d'opérations de tenues de registre et d'enregistrement des affaires destinées à assurer un meilleur suivi et à prendre connaissance des affaires en retard. Celles-ci devaient normalement être traitées dans un délai de quinze jours. Hormis la présidence du conseil

16. Arch. nat., $\mathrm{F}^{13} 325^{\mathrm{B}}$, Projet de règlement pour la tenue des séances du conseil des bâtiments civils et la surveillance des travaux conformément à l'organisation du ministre et aux arrêtés pris par le Conseil, [après le 2 ou 3 brumaire an VII (23 ou 24 octobre 1798)].

17. Arch. nat., $\mathrm{F}^{13}$ 201, Rapport de Heurtier au ministre de l'Intérieur du 19 mai 1807. 
des bâtiments civils par le ministre ou le directeur des Travaux de Paris, les méthodes d'examen ne changèrent pas fondamentalement.

L'organisation de 1838 reproduisait en grande partie le même ordre de travail. Toutefois, elle apportait des précisions sur la production des rapports en indiquant que ceux-ci devaient se terminer par un résumé complet des propositions des rapporteurs et qu'ils devaient être mis au net et déposés au secrétariat le jour de la séance qui précédait celle où ils devaient être lus, de sorte que les membres pussent en prendre connaissance. On comprend donc qu'une partie du travail, et peut-être de la discussion, était conduite en amont des séances.

\section{La forme des avis}

Jusqu'en 1840 environ les procès-verbaux présentent une copie du rapport et l'enregistrement de l'avis du conseil des bâtiments civils. En conséquence de quoi, on ne conserve généralement que le résultat final de la délibération du Conseil et le rapport qui la provoque, les éléments de la discussion étaient donc gommés. Ponctuellement, quelques-uns seulement laissent transparaître des désaccords profonds ou des hésitations. Il n'est pas vraiment possible de savoir plus en détail comment se déroulaient les débats, les modes de résolution des conflits, ou plus précisément la manière dont l'avis était formulé pour être couché par écrit. Si des conflits survinrent, ceux-ci ne donnèrent généralement pas lieu à la production de traces écrites pour la période étudiée. Des procès-verbaux, on ne peut que se contenter de percevoir le décalage fréquent entre le rapport et l'avis.

En 1853, probablement à la faveur de l'entrée de nouveaux membres qui avait rompu la routine de l'institution, un conflit ouvert éclata entre le secrétaire et d'autres membres. Il fut suffisamment grave pour que le vice-président se sente obligé de solliciter la démission du secrétaire et que sa place fut attribuée à un employé du bureau des bâtiments civils ${ }^{18}$. Nous n'avons pas pu identifier l'objet précis du litige. Cependant, les pièces conservées sont relativement éloquentes sur l'importance de cette place dans les débats. Le fait qu'une même personne soit à la fois membre du Conseil et secrétaire était jugé fâcheux au motif que «le secrétaire ne se trouve pas, vis-à-vis du Conseil dans une position de dépendance qui assure toute liberté dans les discussions ${ }^{19}$ ".

Prenant acte de cette décision, la défense de Charles-Pierre Gourlier (1786-1857), qui était ici concerné, n'en était pas moins intéressante. D’abord il faisait remarquer que la place lui avait été rendue à la demande du directeur-président, car on s'était rendu à l'évidence qu'il était nécessaire que ces fonctions soient occupées par un architecte. Pour le secrétaire, c'était la nature de la fonction même qui l'exposait aux critiques.

18. Arch. nat., $\mathrm{F}^{21} 1836$, Lettre d'Auguste Caristie, vice-président du Conseil au ministre de l'Intérieur du 29 mars 1853.

19. Ibid. 
Mais, obligé à chaque séance de noter les opinions diverses et de formuler une opinion presque toujours contraire à celui de l'un ou l'autre des membres, un secrétaire est exposé à les froisser ainsi tour à tour et à amasser contre lui une masse de mécontentements même involontaires ${ }^{20}$.

Le secrétaire précisait aussi, comment dans certains cas on avait cherché à rendre compte des divergences dans la rédaction des avis.

J'ose croise, dans les nombreux rapports, procès-verbaux et avis rédigés par moi, avoir apporté non le talent, mais tous les soins nécessaires, et de plus toute abnégation d'opinion personnelle dans un certain nombre d'avis contraires à ma manière de voir ou dans lesquels, pour quelques affaires importantes, le Conseil a cru devoir exposer l'avis de la majorité et de la minoritée ${ }^{11}$.

Par chance, pour les années 1839-1841, parallèlement aux séries de procès-verbaux, des notes prises au Conseil ainsi que des minutes des séances sont conservées ${ }^{22}$. Elles permettent, en partie, d'étudier la formation des avis produits par le conseil des bâtiments civils et de nous éclairer sur la marche de la délibération. L'intérêt principal de ces documents c'est qu'à la différence des procès-verbaux, ils notent directement les avis des différents membres et le déroulé de la discussion. Dans leur tour de parole, les membres pouvaient faire part d'appréciations assez étendues sur des principes de doctrine. Pour les affaires que nous avons étudiées, les avis pouvaient être très divergents et l'on comprend bien la difficulté que pouvait rencontrer le secrétaire pour la rédaction du compte rendu et la formulation de l'avis. Si les minutes font état d'un essai de formulation de l'avis en séance, tout montre que sa rédaction finale était l'objet d'une recomposition qui pouvait parfois se révéler très importante.

En dépit des différences de statut entre les rapporteurs ou les inspecteurs généraux et les membres du Conseil, et même si l'on entraperçoit une influence plus ou moins grande de certains membres, il semble que les relations au sein de l'institution étaient plutôt confraternelles et d'abord marquées par un certain respect vis-àvis de la carrière des plus anciens.

En commandant l'accès à l'ensemble de la commande publique - la plus rémunératrice et la plus importante pour la notoriété de l'architecte - et par la réglementation du processus de production architectural, le Conseil et l'administration des bâtiments civils fut à l'origine, selon le mot de Georges Teyssot, d'un véritable "système des bâtiments civils » dont le rôle fut sans doute plus déterminant encore

20. Arch. nat., $\mathrm{F}^{21}$ 1836, Lettre de Charles-Pierre Gourlier au ministre de l'Intérieur, s.d. [c. avril 1853]. 21. Ibid.

22. Les avis au Conseil Arch. nat., $\mathrm{F}^{21} 2537$ à 2539 et les notes prises aux séances du Conseil $\mathrm{F}^{21} 2540$ à 25421 ne sont conservées que pour quelques années (respectivement 1839 à 1844 et 1838 à 1856) mais peuvent être mis en relation avec la série de Procès-verbaux. Nous procédons actuellement à l'étude détaillée de ce corpus. 
que celui des Beaux-Arts dans la définition de la pratique architecturale au cours de la première moitié du XIX siècle $^{23}$. Bien loin de l'institution académique souvent décrite, nous avons déjà montré que l'action du conseil des bâtiments civils se caractérisait plutôt par un certain pragmatisme et un éclectisme a la fois ouvert a l'innovation architecturale et stylistique et préoccupé par la défense de la profession d'architecte $^{24}$. Toutefois, si la création de l'institution se justifiait principalement par la nécessité de garantir la responsabilité ministérielle et de légitimer la commande publique, le caractère normatif de son activité ne fait aucun doute car par l'intermédiaire de ses avis, le Conseil rejetait, rectifiait ou approuvait des projets architecturaux.

La richesse de la source constituée par la série des procès-verbaux du conseil des bâtiments civils nous paraît donc fondamentale afin de pouvoir observer les inflexions du discours architectural dans la première moitié du siècle. Au travers de ce commentaire d'architecture contenu dans les 30000 procès-verbaux du conseil des bâtiments civils jusqu'en 1848, on peut dire que s'élabore une véritable théorie de l'architecture publique. Or, cette théorie s'énonce de manière discursive dans le contexte contingent, à chaque fois renouvelé, de l'examen des projets. Il nous semble en effet que les rapports entre la théorie et la pratique ne sauraient être envisagés de manière trop figée et qu'il n'est pas possible de cantonner les manifestations de la théorie aux seuls grands ouvrages imprimés qui mettent en ordre les savoirs. À cet égard, depuis la fondation de l'Académie royale d'architecture, l'examen des projets concrets et la pragmatique du chantier ont toujours occupé une place de choix dans l'élaboration de la doctrine en France ${ }^{25}$. Or pour la période concernée, l'Académie des Beaux-Arts qui prit la suite de la section des Beaux-Arts de l'Institut n'était plus que très marginalement consultée sur les projets architecturaux. Le pouvoir de légiférer sur le projet ayant été transféré au conseil des bâtiments civils, ce pan fondamental dans la constitution de la doctrine lui échappait désormais ${ }^{26}$.

En somme, il nous semble que le formidable corpus textuel que forment les procèsverbaux du conseil des bâtiments civils dans la première moitié du XIX ${ }^{\mathrm{e}}$ siècle devrait être un point d'observation privilégié pour étudier l'évolution du rapport à la conception architecturale et les transformations du discours normatif en architecture. Si pour Jean-Pierre Epron, l'éclectisme se manifeste d'abord en architecture par une nouvelle attitude à l'égard du projet qui s'observe notamment dans les

23. Georges Teyssot, "Types, programmes et régularité. La diffusion des principes architecturaux au sein du conseil des bâtiments civils sous le Consulat et l'Empire ", Villes et territoire pendant la période napoléonienne (France et Italie), actes du colloque de Rome, 3 au 5 mai 1984, Publications de l'École française de Rome, 96, Rome, École française de Rome, 1987, p. 231-245.

24. Emmanuel Château-Dutier, op. cit.

25. Robert Carvais, Rapport final du projet Desgodets, ANR-07-CORP-017-01, Paris.

26. Jean-Michel Leniaud, "L'édition des procès-verbaux de l'Académie des Beaux-Arts : une source pour l'étude du grand prix de Rome ", Romantisme, vol. 153, nº 3, 2011. Doi :10.3917/rom.153.0117 
pratiques et les discours des institutions architecturales telles que la Société d'architecture créée en 1840 , ou le conseil des bâtiments civils ${ }^{27}$, cet éclectisme devrait pouvoir être mis à jour à travers l'étude diachronique du texte de ses délibérations.

Emmanuel CHÂTEAU-DUTIER

Université de Montréal

27. Jean-Pierre Epron, Comprendre l'éclectisme, Paris, Norma, 1997. 